\title{
The human otitis media with effusion: a numerical-based study
}

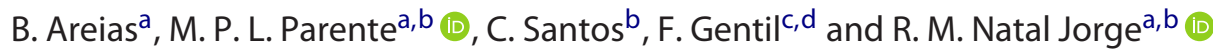 \\ ${ }^{a}$ INEGI, Institute of Science and Innovation in Mechanical and Industrial Engineering, Porto, Portugal; ${ }^{b}$ FEUP, Faculty of Engineering, University \\ of Porto, Porto, Portugal; ${ }^{C}$ ESS, Escola Superior de Saúde, P. Porto, Porto, Portugal; ${ }^{\mathrm{d}}$ Clínica ORL - Dr. Eurico de Almeida, Porto, Portugal
}

\begin{abstract}
Otitis media is a group of inflammatory diseases of the middle ear. Acute otitis media and otitis media with effusion (OME) are its two main types of manifestation. Otitis media is common in children and can result in structural alterations in the middle ear which will lead to hearing losses. This work studies the effects of an OME on the sound transmission from the external auditory meatus to the inner ear. The finite element method was applied on the present biomechanical study. The numerical model used in this work was built based on the geometrical information obtained from The visible ear project. The present work explains the mechanisms by which the presence of fluid in the middle ear affects hearing by calculating the magnitude, phase and reduction of the normalized umbo velocity and also the magnitude and phase of the normalized stapes velocity. A sound pressure level of $90 \mathrm{~dB}$ SPL was applied at the tympanic membrane. The harmonic analysis was performed with the auditory frequency varying from $100 \mathrm{~Hz}$ to $10 \mathrm{kHz}$. A decrease in the response of the normalized umbo and stapes velocity as the tympanic cavity was filled with fluid was obtained. The decrease was more accentuated at the umbo.
\end{abstract}

\section{KEYWORDS}

Otitis media; middle ear; tympanic cavity;

biomechanics

\section{Introduction}

Otitis media is a common disease characterized by the inflammation of the middle ear mucosal, with or without an effusion (Swarts et al. 2014). Often begins when an infection that causes sore throats, colds or other respiratory problems which then spreads to the middle ear, through the Eustachian tube. Otitis media with effusion (OME) is defined as the presence of fluid in the middle ear without signs or symptoms of acute ear infection. It generally occurs spontaneously, due to a poor Eustachian tube function or as an inflammatory response, following an acute otitis media (American Academy of Family Physicians 2004). The Eustachian tube has been described with three main functions: protection, pressure regulation and clearance (Douglas Swarts \& Bluestone 2003). This tube is normally closed and can be opened by the contractions of the tensor veli palatini muscle during swallowing or passively opened by increasing the middle ear or nasopharyngeal pressure to supra-ambient levels (Doyle et al. 2013; Swarts et al. 2014). Experiments in monkeys and other animal species demonstrated that an inability to open the Eustachian tube causes the successive development of negative middle ear pressures, mucosal inflammation and effusion accumulation in the normally air-filled tympanic cavity (TC) (Doyle 1984). Otitis media is the primary cause of hearing losses in the population and is associated with other complications such as balance disturbances and speech and language development delays in children (Doyle et al. 2013). In infants, the Eustachian tube is shorter, wider and more horizontal than in adults, so the protective function of the tube may be lower in this age group (Daniel et al. 1988). The fluid present in the middle ear can be serous, mucoid or glue-like and the TC may be partly or completely filled with fluid. Fluid in the middle ear has been associated with a conductive hearing loss of 25 to $30 \mathrm{~dB}$ (Ravicz et al. 2004). The frequent presence of fluid in the TC results in a decreased mobility of the tympanic membrane and serves as a barrier to sound conduction. Approximately, $90 \%$ of children report episodes of OME at some time before the school age (American Academy of Family Physicians 2004; Valente et al. 2010; Atkinson et al. 2015). There are several experimental studies about otitis media in children. Numerical studies are important to understand the alterations caused by this disease and thus contribute to the development of more effective treatment techniques. The study reported here intends to show the influence of OME in the normal functioning of the human ear. A 3D model of the human ear was developed and the finite element method was applied to obtain the responses in the umbo and in the stapes footplate. A comparison of the responses with previously published data in the literature 
to validate the results obtained, was also performed. The software Abaqus Standard (Hibbit et al. 2004) was used for the various analyses. A direct-solution steady-state dynamic analysis was performed to obtain the frequency response. This type of analysis provides the steady-state displacements magnitude and phase angle of the response of the system due to harmonic excitation, at a given frequency. The analysis is done by applying the loading at a series of different frequencies.

\section{Materials and methods}

\subsection{Finite element analysis}

The structural response was computed at discrete excitation frequencies by solving a set of coupled matrix equations using complex algebra (Jazar 2012). In direct frequency response analysis, the equation of motion to a harmonic excitation is given by,

$$
[M]\{\ddot{u}(t)\}+[C]\{\dot{u}(t)\}+[K]\{u(t)\}=\{P(\omega)\} e^{i \omega t}
$$

where $M$ is the mass matrix, $C$ is the damping matrix, $K$ is the stiffness matrix and $P(\omega)$ is an external harmonic load with frequency $\omega$.

A harmonic perturbation, $p_{s}(t)$, with amplitude $P$ and frequency $\omega$ can be described by a sinusoidal function. This sinusoidal function may be equivalently written in terms of the complex exponential $e^{i \omega t}$.

$$
p_{s}(t)=P(\cos (\omega t)+i \sin (\omega t))=P e^{i \omega t}
$$

The particular solution to the differential equation, $u(t)$ represent the stationary response due to the harmonic perturbation.

$$
u(t)=\bar{U}(\omega) e^{i \omega t}
$$

where the $\bar{U}(j \omega)$ is the complex displacement vector. The first and second time derivatives of the complex displacement are given by,

$$
\begin{aligned}
& \dot{u}(t)=j \omega \bar{U}(\omega) e^{i \omega t} \\
& \ddot{u}(t)=-\omega^{2} \bar{U}(\omega) e^{i \omega t}
\end{aligned}
$$

Substituting the particular solution, the first and the second time derivatives in the equation of motion, the equation becomes,

$$
\begin{aligned}
& {[M]\left\{-\omega^{2} \bar{U}(\omega) e^{i \omega t}\right\}+[C]\left\{j \omega \bar{U}(\omega) e^{i \omega t}\right\}} \\
& +[K]\left\{\bar{U}(\omega) e^{i \omega t}\right\}=\{P(\omega)\} e^{i \omega t}
\end{aligned}
$$

Dividing all terms by $e^{i \omega t}$,

$$
\left[-\omega^{2} M+j \omega C+K\right]\{\bar{U}(\omega)\}=\{P(\omega)\}
$$

The constitutive behaviour of the fluid in the cochlea and in the TC is assumed to be inviscid, linear and compressible, being described by the following equation.

$$
p_{f}=-B_{f} \frac{\partial}{\partial x} u^{f}
$$

where $p_{f}$ is the excess pressure in the fluid (the relative pressure), $x$ is the spatial position of the fluid particle, $u$ is the fluid particle displacement and $B_{f}$ is the bulk modulus of the fluid (Hibbit et al. 2004).

\subsection{Geometrical model}

The geometrical model was obtained from the project The Visible Ear (Wang et al. 2007). In this project, the anatomical data of a temporal bone of an 85 years old woman without pathologies was obtained by using a cryosectioning procedure. A set of high quality images of a frozen temporal bone was acquired. The slice thickness was $25 \mu \mathrm{m}$ and high resolution images were captured every $50 \mu \mathrm{m}$. A total of 605 images with 24 bits RGB and a resolution of $50 \mu$ mpixel $^{-1}$ was obtained. A total of 26 different structures of the human ear were identified through manual segmentation of the entire set of images. Using the segmented structures, the finite element mesh shown in Figure 1 was constructed, including the tympanic membrane (TM), the ossicular bones malleus (M), incus (I) and stapes (S), the cochlea (C), part of the jaw and temporal bone (TB) and the air in TC. The insertion of the tympanic annulus (TA) into the temporal bone (Prendergast et al. 1999) was modelled as shown in Figure 1. The cochlea was simulated as a single scale and no vestibular nor tympanic scale were considered in the model. The acoustic elements of the cochlea and of the TC were attached to the continuum elements of the temporal bone, through the TIE command available in Abaqus Standard (Hibbit et al. 2004). This option allows to impose the acoustic-mechanical interactions between pairs of surfaces. The model also includes two tendons (stapedius tendon and tensor tympani tendon), six ligaments (superior malleolar ligament; lateral malleolar ligament; anterior malleolar ligament; superior incudal ligament; posterior incudal ligament; stapedius annular ligament) and the incudomalleolar and incudostapedial joints. The model of the ear was oriented in space following the proposed orientation by Ravicz et al. (2004). The orientation of the model is important since it will affect the way as the fluid fills the cavity. The ear model was orientated to coincide with a seated position. 


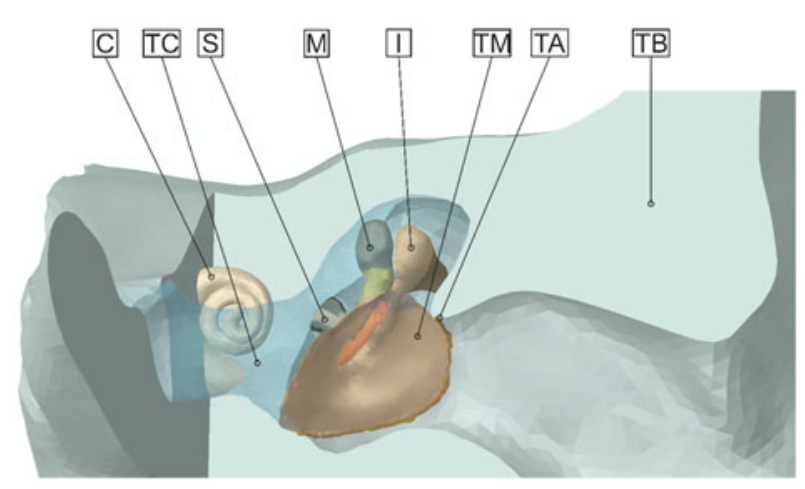

Figure 1. Finite element model.

\subsection{Material properties}

The mechanical properties attributed to the structural components of the model, ligaments and tendons, and acoustic mediums are shown in Tables 1-3, respectively. The mechanical properties presented in Tables 1 and 2 were based on the work of Sun et al. (2002) and Gentil et al. (2011). Linear elastic properties were incorporated in all parts of the 3D finite element model. The Poisson's ratio was assumed to be 0.3 for all parts of the ear (Sun et al. 2002; Gentil 2008; Areias 2014). The Raleigh's proportional damping with the coefficients $\alpha=0 \mathrm{~s}^{-1}$ and $\beta=0.0001 \mathrm{~s}$ was introduced in the ear components and in the ligaments and tendons (Lee et al. 2006; Liu et al. 2009). The TM was considered as being composed by one layer (Ferris \& Prendergast 2000) and was divided into pars tensa with orthotropic properties and pars flaccida with isotropic properties. The air and the fluid inside the TC and the fluid inside the cochlea were modelled with acoustic elements. The acoustical properties (density, $\rho_{f}$ and bulk modulus, $B_{f}$ ) of the acoustic mediums are shown in the Table 3.

To analyse the viscosity parameter, (Ravicz et al. 2004), using an experimental set-up, varied the viscosity of the fluid that was introduced into TC from $1 \times 10^{-6}$ (saline solution) to $1.2 \times 10^{-2} \mathrm{~m}^{2} \mathrm{~s}$ (silicone solution). Ravicz results showed a small change in the normalized umbo velocity response due to this viscosity variation. On the present work, the fluid was assumed as inviscid. In Abaqus Standard, a steady-state dynamics procedure does not take account the viscosity parameter.

Inadequate mesh refinement is one of the most common sources of difficulties in acoustic and vibration analysis. For reasonable accuracy, at least six representative internodal intervals $\left(n_{\min } \geq 6\right)$ of the acoustic mesh should fit into the shortest acoustic wavelength present in the analysis $\left(f_{\max }=10 \mathrm{kHz}\right)$. The internodal interval is defined as the distance between the two nearest nodes in an element. The maximum linear element length $\left(L_{\max }\right)$ is given by,

$$
L_{\max }=\frac{c_{f}}{n_{\min } \times f_{\max }}
$$

where $c_{f}$ is the speed of the sound in the medium and is achieved through the bulk modulus $B_{f}$, and the density $\rho_{f}$.

$$
c_{f}=\sqrt{\frac{B_{f}}{\rho_{f}}}
$$

Thus, the maximum linear element length for the acoustic elements, based on the properties listed in Table 3 , is given by,

$$
\begin{aligned}
L_{\text {max TC air }} & =\frac{\frac{1.01 \times 10^{5}}{1.164}}{6 \times 10,000}=0.0049 \mathrm{~m}=4.9 \mathrm{~mm} \\
L_{\max \text { TC fluid }} & =\frac{\frac{2.2 \times 10^{9}}{1000}}{6 \times 10,000}=0.0247 \mathrm{~m}=24.7 \mathrm{~mm} \\
L_{\text {max Cochlea }} & =\frac{\frac{2.2 \times 10^{9}}{1000}}{6 \times 10,000}=0.0247 \mathrm{~m}=24.7 \mathrm{~mm}
\end{aligned}
$$

The mean acoustic elements length of the TC and the cochlea was 0.4 and $0.365 \mathrm{~mm}$, respectively, thus satisfying the previous requirement.

\subsection{Boundary conditions and model set up}

In order to study the effects of the presence of fluid on the sound transmission due to OME, the TC was filled with different levels of fluid. Figure 2 shows the different fluid levels in the TC used in the numerical analysis of OME: A - dry TC (normal situation); B - 30\% of the TC with fluid; C $-60 \%$ of the TC with fluid; D $-100 \%$ of the TC with fluid. The properties of the fluid inside the TC were assumed similar to the water properties (Table 3). The Eustachian tube was considered closed on the connection with the nasopharynx. The part of the temporal bone and jaw sectioned were fixed in all degrees of freedom and a sound pressure level of $90 \mathrm{~dB}$ SPL was applied to the TM. The model was discretized with 140,441 nodes and 703,303 elements, of which 578,774 were C3D4 elements, 124,522 were acoustic AC3D4 elements and 7 were truss T3D2 elements. The tendons and ligaments were simulated using truss T3D2 elements. The acoustic components (cochlea, fluid and air in the TC) were meshed with acoustic AC3D4 elements and the remaining parts of the ear with tetrahedral C3D4 elements. The TC was discretized with 83,230 linear tetrahedral acoustic (AC3D4) elements. The C3D4 are continuum three-dimensional tetrahedral elements and first-order with four nodes and one integration point 
Table 1. Material properties for the ear components.

\begin{tabular}{lcc}
\hline Ear components & Density $\rho\left[\mathrm{kg} \mathrm{m}^{-3}\right]$ & Young's modulus $E[\mathrm{~Pa}]$ \\
\hline Tympanic membrane (TM) & $1.2 \times 10^{3}$ & Pars tensa \\
& & $3.2 \times 10^{7}(r)^{\mathrm{a}}$ \\
$2.0 \times 10^{7}(\theta)^{\mathrm{b}}$ \\
Pars flaccida \\
\\
& & $1.0 \times 10^{7}$ \\
& & \\
Malleus (M) & $2.55 \times 10^{3}$ (Head) & $1.41 \times 10^{10}$ \\
& $4.53 \times 10^{3}$ (Neck) & \\
Incus (I) & $3.70 \times 10^{3}$ (Handle) & \\
& $2.36 \times 10^{3}$ (Body) & \\
& $2.26 \times 10^{3}($ Short process) & $1.41 \times 10^{10}$ \\
Stapes (S) & $5.08 \times 10^{3}$ (Long process) & \\
Incudomalleolar Joint & $2.2 \times 10^{3}$ & $1.41 \times 10^{10}$ \\
Incudostapedial Joint & $3.2 \times 10^{3}$ & $1.41 \times 10^{10}$ \\
Temporal bone (TB) and Jaw & $1.2 \times 10^{3}$ & $6.0 \times 10^{5}$ \\
Round window & $2.0 \times 10^{3}$ & $1.41 \times 10^{10}$ \\
\hline
\end{tabular}

a Radial direction.

${ }^{\mathrm{b}}$ Circunferencial direction.

Table 2. Material properties for the ligaments and tendons.

\begin{tabular}{|c|c|c|c|}
\hline Ligaments and tendons & Density $\rho\left[\mathrm{kg} \mathrm{m}^{-3}\right]$ & Young's modulus $E[\mathrm{~Pa}]$ & Damping \\
\hline Superior malleolar ligament & $2.5 \times 10^{3}$ & $4.9 \times 10^{4}$ & \\
\hline Lateral malleolar ligament & $2.5 \times 10^{3}$ & $6.7 \times 10^{4}$ & \\
\hline Anterior malleolar ligament & $2.5 \times 10^{3}$ & $2.1 \times 10^{6}$ & \\
\hline Superior incudal ligament & $2.5 \times 10^{3}$ & $4.9 \times 10^{4}$ & $\alpha=0 \mathrm{~s}^{-1}$ \\
\hline Posterior incudal ligament & $2.5 \times 10^{3}$ & $6.5 \times 10^{5}$ & $\beta=0.0001 \mathrm{~s}$ \\
\hline Tensor tympani tendon & $2.5 \times 10^{3}$ & $2.6 \times 10^{6}$ & \\
\hline Stapedius tendon & $2.5 \times 10^{3}$ & $5.2 \times 10^{5}$ & \\
\hline Tympanic annulus (TA) & $1.2 \times 10^{3}$ & $6.0 \times 10^{4}$ & \\
\hline Stapedius annular ligament (SA) & $2.5 \times 10^{3}$ & $2.0 \times 10^{4}$ & \\
\hline
\end{tabular}

Table 3. Acoustical properties.

\begin{tabular}{llc}
\hline Acoustic medium & $\begin{array}{l}\text { Density } \\
\rho_{f}\left[\mathrm{~kg} \mathrm{~m}^{-3}\right]\end{array}$ & $\begin{array}{l}\text { Bulk modulus } \\
B_{f}[\mathrm{~Pa}]\end{array}$ \\
\hline TC air & 1.164 & $1.01 \times 10^{5}$ \\
TC fluid & 1000 & $2.2 \times 10^{9}$ \\
Cochlear fluid (C) & 1000 & $2.2 \times 10^{9}$ \\
\hline
\end{tabular}

(reduced integration). The AC3D4 are acoustic continuum three-dimensional elements. They are also firstorder tetrahedral elements with four nodes but with four integration points (full integration), having only one degree of freedom (pressure degree of freedom). The T3D2 truss elements are three dimensional, 2-node truss elements with linear displacements.

\section{Results}

In the developed model, the influence of OME on the normal ear was studied. Figures 3 and 4 show, respectively, the magnitude, $|\hat{V}|_{\text {umbo }}$ and phase angle, $\measuredangle \hat{V}_{\text {umbo }}$

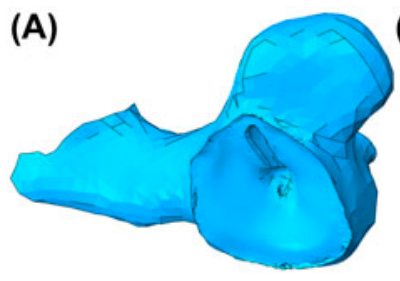

(B)
(C)

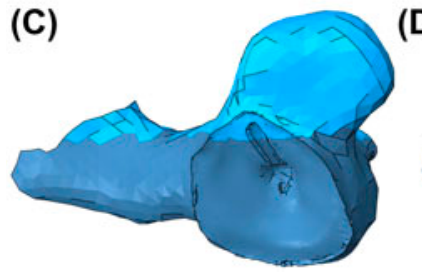

(D)

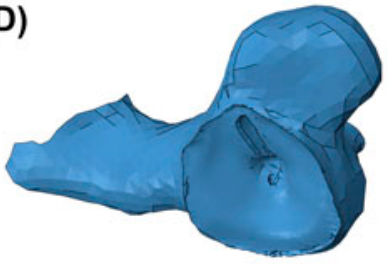

Figure 2. Fluid levels in the tympanic cavity.

of the normalized umbo velocity. Since the umbo stapes velocity varies linearly with the pressure applied on the $\mathrm{TM}$, the umbo velocity can be normalized. The normalized velocity is calculated through the ratio of the umbo velocity, $V$, and the sound pressure applied to the TM, $P$, 


$$
\hat{V}=\frac{V}{P}
$$

since $\hat{V}$ is a complex number, the magnitude $|\hat{V}|$ and the phase angle $\measuredangle \hat{V}$ of $\hat{V}$ were obtained.

The frequency range in consideration varies between $100 \mathrm{~Hz}$ and $10 \mathrm{kHz}$. A sound pressure level of $90 \mathrm{~dB}$ SPL was applied on the TM for all levels of fluid on the TC, $0,30,60$ and $100 \%$ fluid-filled. The numerical results achieved were compared with the experimental data of Ravicz et al. (2004) and Gan et al. (2006). In his work, Ravicz et al. (2004) published experimental results of nine normal human temporal bones. The ages of the individuals ranged from 54 to 75 years, with a mean of 67 years. Absence of otology disease on review of the medical chart and by visual inspection of the external auditory meatus, middle ear and mastoid using and operating microscope was verified. The middle ear air volume in the nine human temporal bones varied between 600 and $1200 \mu \mathrm{L}$. In the present numerical model, the middle ear has a volume of $476 \mu \mathrm{L}$.

Gan et al. (2006) used eleven fresh-frozen cadaver temporal bones in its study. The ages of individual donors ranged from 50 to 93 with a mean of 71 years. Absence of overt pathology was confirmed. Pure tone sound signals of $90 \mathrm{~dB}$ sound pressure level (SPL) were delivered to the ear canal near the TM by an inserted earphone. Peakto-peak displacement of the TM measured from seven temporal bones with intact cochlea when saline solution was introduced into the TC from 0 to $600 \mu \mathrm{L}$ were obtained.

The results obtained are close to the experimental data of Ravicz et al. (2004) and Gan et al. (2006). In the normal situation of the TC, considering the cavity filled with air and the Eustachian tube closed, the $|\hat{V}|_{\max \text { umbo }}$ is obtained near to $890 \mathrm{~Hz}$. Ravicz et al. (2004) obtained the peak at $2100 \mathrm{~Hz}$ for one of the temporal bones studied (temporal bone 27A in its paper), the results are shown in Figure 3. Considering the average values of the temporal bones analysed by the same author, the frequency with maximum umbo magnitude was $1200 \mathrm{~Hz}$. A slope near 1 on a $\log -\log$ scale below $890 \mathrm{~Hz}\left(|\hat{V}|_{\text {max umbo }}\right)$ was obtained for the normal condition in the TC. On the other hand, above this value, the slope was -1 .

In the work of Gan et al. (2006), the peak of $|\hat{V}|_{\text {umbo }}$ is located at $1300 \mathrm{~Hz}$. The response does not decrease after the peak; a small increase at $4200 \mathrm{~Hz}$ was verified. At low frequencies, the results obtained in the present study are very close to the ones obtained by Ravicz et al. (2004) and are slightly higher than the results achieved by Gan et al. (2006).

Some authors, such as Gan and Wang (2007) proposed the fact that the mechanical properties of the soft tissue may change when exposed to a liquid. To obtain a good agreement with the experimental data, in the work of Gan and Wang (2007) the Young's modulus of the incudostapedial joint was modified, according to the level of the fluid (it was increased by a factor of 12.5 when the fluid volume in the TC increased from 50 to $100 \%)$. The present study does not take into account the alterations caused by the fluid in the geometry and material properties of the middle ear structure, which could be the cause of the differences between the present numerical results and the experimental data. Nevertheless, the effect of the fluid does not have yet a clear justification, as refereed by Gan and Wang (2007).

By increasing the amount of fluid in the TC, a sharper drop of $|\hat{V}|_{\text {umbo }}$ at high frequencies was verified. There was also a substantial reduction in the frequency where $|\hat{V}|_{\max }$ umbo was reached. When the TC contains 30 and $60 \%$ of fluid, the $|\hat{V}|_{\max \text { umbo }}$ was obtained at 700 and $550 \mathrm{~Hz}$, respectively. For frequencies below $550 \mathrm{~Hz}$, $|\hat{V}|_{\text {umbo }}$ remained unchanged between the normal TC, and the TC with 30 and $60 \%$ of fluid. When the TC was fully filled with fluid, a decrease was observed in $|\hat{V}|_{\text {umbo }}$ over the whole frequency band studied. Contrarily to what happened with the TC partly filled, when the TC was completely filled with fluid, there was a decrease in the $|\hat{V}|_{\text {umbo }}$ for frequencies below $550 \mathrm{~Hz}$. In the work of Ravicz et al. (2004) and Gan et al. (2006) this decrease was also verified.

In the normal situation (TC filled with air), the $\measuredangle \hat{V}_{\text {umbo }}$ numerical curve is similar to the experimental data of Ravicz et al. (2004). When increasing the amount of fluid in the TC, a reduction in the phase of the normalized umbo velocity between 400 and $2000 \mathrm{~Hz}$ was observed. When the TC was fully filled with fluid, a decrease in the $\measuredangle \hat{V}_{\text {umbo }}$ extends to higher frequencies, up to $4000 \mathrm{~Hz}$. A phase shift of $180^{\circ}$ at $4500 \mathrm{~Hz}$ was verified, accompanied by an exchange from negative to positive in the slope of the $|\hat{V}|_{\text {umbo }}$.

To simplify the comparison of $|\hat{V}|_{\text {umbo }}$ between the normal situation and the situations with a presence of fluid, the reduction in the magnitude of the normalized umbo velocity, $\Delta V_{\text {umbo }}$ was calculated (Figure 5). The $\Delta V_{\text {umbo }}$ was calculated by the ratio of $|\hat{V}|_{\text {umbo }}$ in the situation with the presence of the fluid in the TC and $|\hat{V}|_{\text {umbo }}$ in the normal situation, expressed in $\mathrm{dB}$. The experimental results of Ravicz et al. (2004) to the same levels of fluid in the TC were also represented. In the Figure 5 , a negative $\Delta V_{\text {umbo }}$ shows a reduction in the magnitude.

As already seen in Figure 3, the decrease in the $|\hat{V}|_{\text {umbo }}$ at low frequencies is only noticeable for those cases in which the TC is completely filled with fluid. A decrease of approximately $25 \mathrm{~dB}$ was obtained for this case. At high frequencies the reduction of the $|\hat{V}|_{\text {umbo }}$ was 


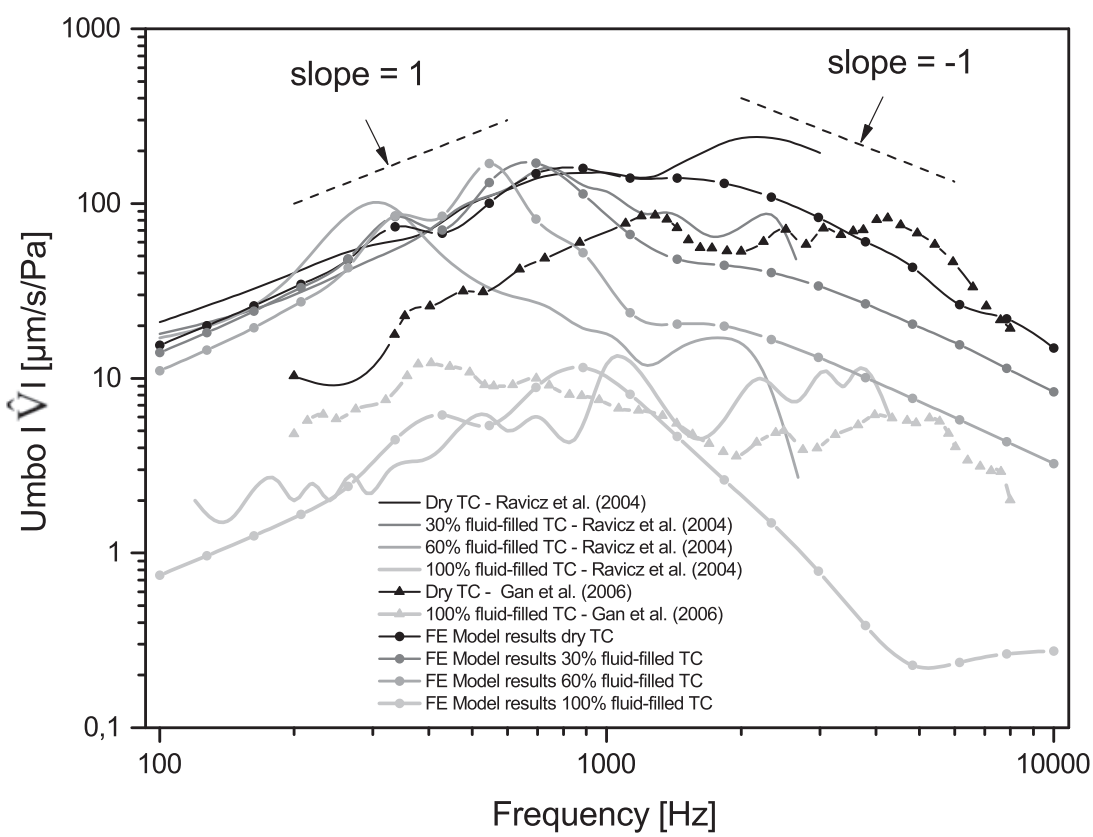

Figure 3. Magnitude of the normalized umbo velocity.

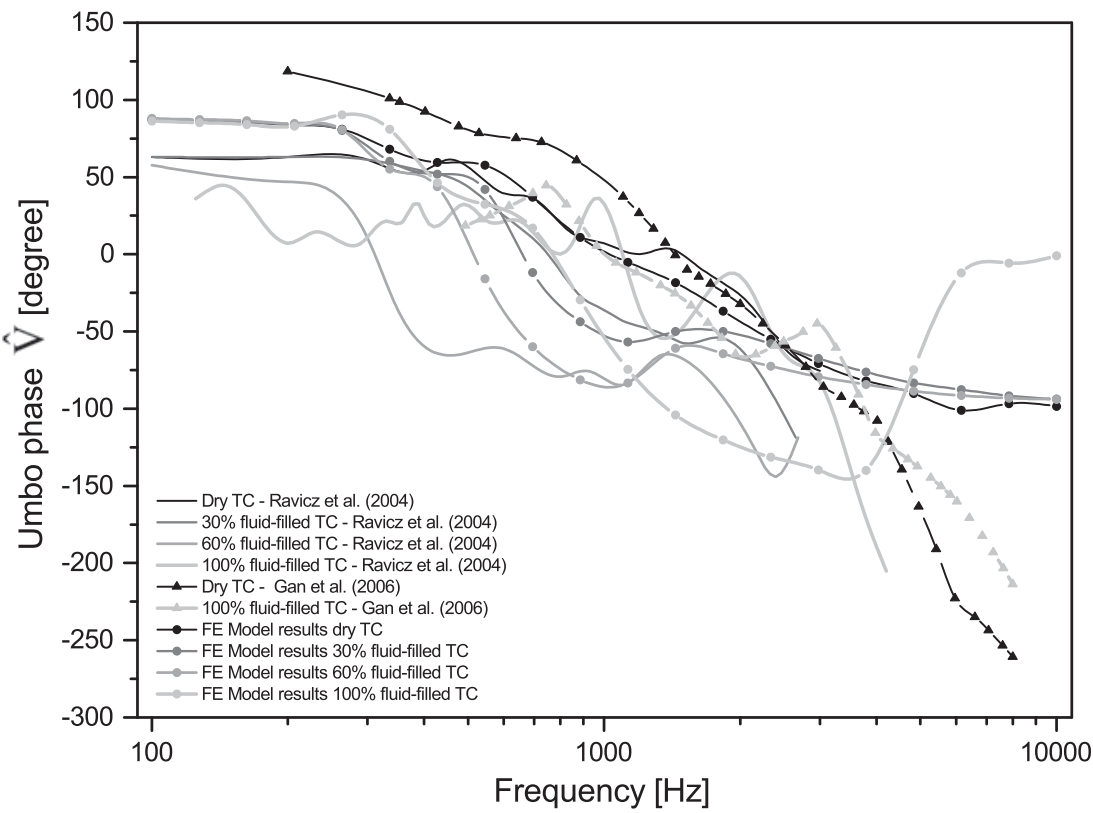

Figure 4. Phase of the normalized umbo velocity.

more gradual, as much as from -5 to $-10 \mathrm{~dB}$ in the case of $30 \%$ of fluid in the TC, from -13 to $-17 \mathrm{~dB}$ in the case of $60 \%$ of fluid and from -25 to $-45 \mathrm{~dB}$ when the TC was fully filled with fluid. A maximum decrease of $45 \mathrm{~dB}$ was obtained near $4800 \mathrm{~Hz}$, when the TC was completely filled with fluid.

Figures 6 and 7 show, respectively, the magnitude $|\hat{V}|_{\text {stapes }}$ and the phase angle $\measuredangle \hat{V}_{\text {stapes }}$ of the normalized stapes velocity. Only for the case of the TC fully filled with fluid, a decrease at low frequencies of $|\hat{V}|_{\text {stapes }}$ was verified. The same occurred with the normalized umbo velocity. At high frequencies the $|\hat{V}|_{\text {stapes }}$ decreases as the TC is filled with fluid, except when the TC was completely and with a presence of $60 \%$ of fluid. In the fully filled case, the $|\hat{V}|_{\text {stapes }}$ increases and exceeds the $|\hat{V}|_{\text {stapes }}$ in the dry situation at $5 \mathrm{kHz}$. The TC works similarly to a rigid pressurized box, and the compression of the fluid caused by the motion of the TM is released in the round 


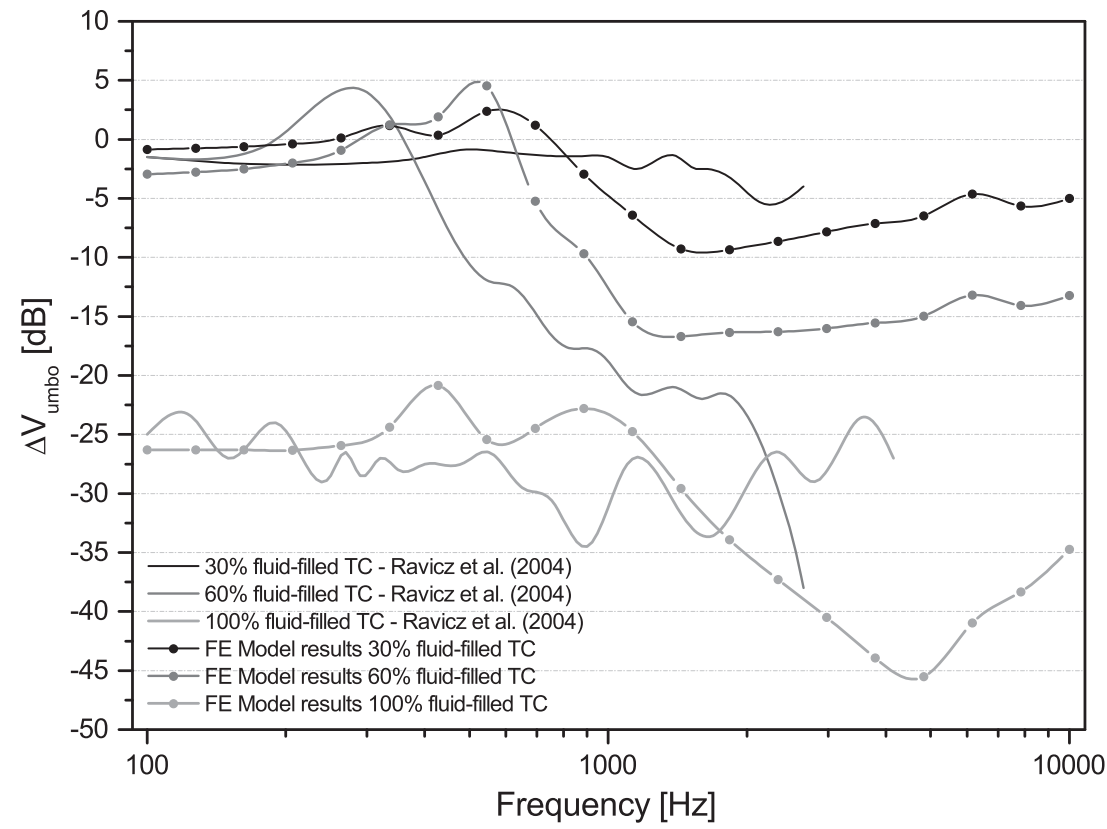

Figure 5. Reduction in magnitude of the normalized umbo velocity.

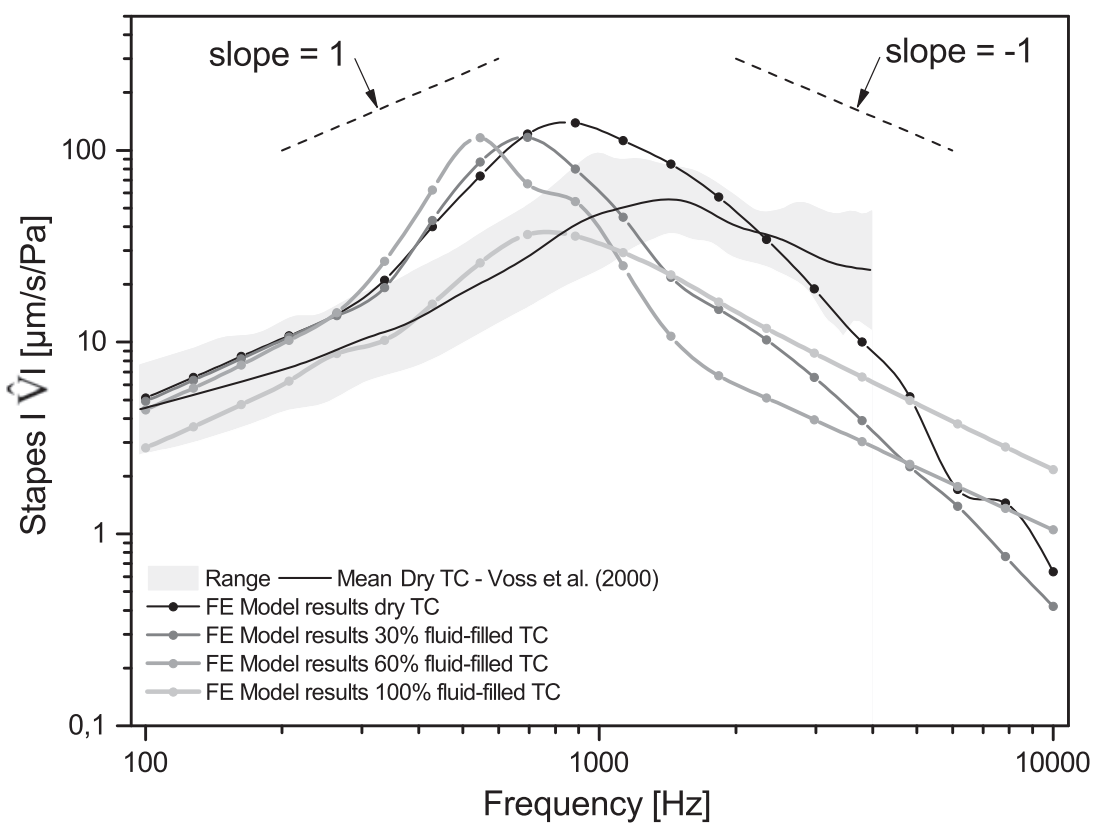

Figure 6. Magnitude of the normalized stapes velocity.

and oval window. Once the bulk modulus of the water is greater than the air, the TC behaviour must be different when is filled with fluid.

A substantial reduction in the frequency of the $|\hat{V}|_{\text {max stapes }}$ was verified as the TC was filled with fluid. A exception when the TC was fully filled with fluid was remarkable. A small decrease in the $|\hat{V}|_{\text {max stapes }}$ between the dry and partly filled situations was verified. The experimental band of Voss et al. (2000) was also included. The curve behaviour is very similar to our results for a dry TC. Voss et al. (2000) results were based on measurements of 18 bones.

Overall, the $\measuredangle \hat{V}_{\text {stapes }}$ tends to decrease up to $3 \mathrm{kHz}$ with the exception when the TC was completely filled. The minimum decrease of the phase angle was obtained for the TC filled with fluid. In this case the phase decreased $180^{\circ}$ between $100 \mathrm{~Hz}$ and $10 \mathrm{kHz}$. A small increase between 300 to $500 \mathrm{~Hz}$ of the $\measuredangle \hat{V}_{\text {stapes }}$ with the TC dry or 


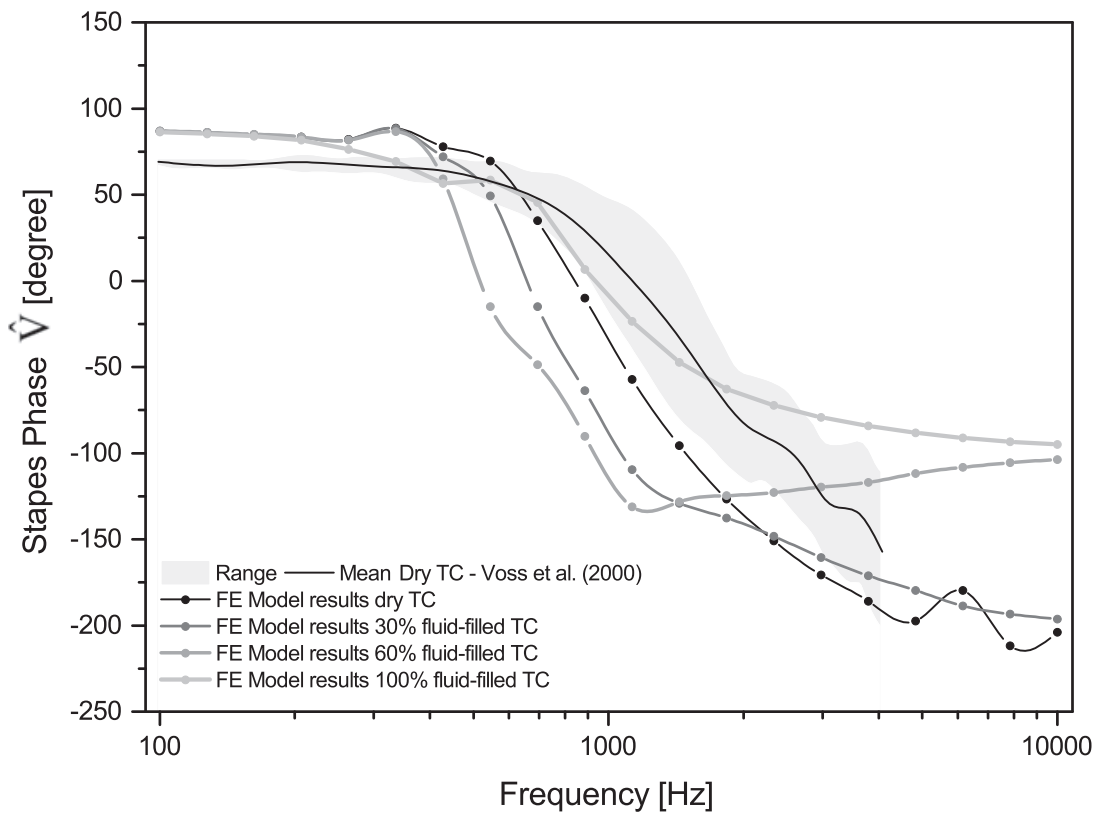

Figure 7. Phase of the normalized stapes velocity.

partly filled was verified, in contrast to the situation with the TC completely filled. The phase angle experimental range at the stapes footplate obtained by Voss et al. (2000) is also in agreement with our outcomes.

\section{Conclusion and discussion}

The numerical results obtained are close to those reported by Ravicz et al. (2004) and Gan et al. (2006). In the normal situation of the TC, filled with air and the Eustachian tube closed, the maximum of the $|\hat{V}|_{\text {umbo }}$ and the $|\hat{V}|_{\text {stapes }}$ were obtained close to $890 \mathrm{~Hz}$. Ravicz et al. (2004) obtained the mean peak at $1200 \mathrm{~Hz}$. A slope near 1 on a log-log scale below $890 \mathrm{~Hz}\left(|\hat{V}|_{\text {max umbo }}\right)$ in the situation with a normal cavity was obtained. On the other hand, above this value the slope was -1 . When increasing the amount of fluid in the TC, a sharper drop of the $|\hat{V}|_{\text {umbo }}$ at the high frequencies was verified. A substantial reduction in the frequency of the $|\hat{V}|_{\max }$ umbo was recorded, as the fluid was introduced into the middle ear. When the TC was completely filled with fluid, there was a decrease observed in the $|\hat{V}|_{\text {umbo }}$ across the frequency range studied. The negative $|\hat{V}|_{\text {umbo }}$ slope is consistent with a mass effect. The mass of the middle ear system becomes more important as fluid is introduced into the middle ear. Overall, the $|\hat{V}|_{\text {stapes }}$ decreases as the TC is filled with fluid, except when the TC is totally filled with fluid and with $60 \%$ of fluid. When the TC is completely filled, the $|\hat{V}|_{\text {stapes }}$ increases and exceeds the $|\hat{V}|_{\text {stapes }}$ in the dry situation at $5 \mathrm{kHz}$. This effect can be attributed to the reduced compressibility of the water when compared with the air. The bulk modulus of the water is greater than the air, leading to higher pressures being developed in the TC when the TC was totally filled with fluid. This occurrence may change the response of the system to some frequencies. A decrease of the $\measuredangle \hat{V}_{\text {stapes }}$ up to $3 \mathrm{kHz}$ in the partly and completely air-filled situation was achieved. The decrease of $\measuredangle \hat{V}_{\text {stapes }}$ was less pronounced when the TC was fully filled with fluid.

Improving the diagnosis and prognosis of ear infections can be of extreme importance, particularly in children. Hearing losses caused by ear infections, although slight and temporary, can have serious consequences in the development of hearing, causing permanent damages to the TM or to other structures in the middle ear. Further analysis of the behaviour of the TM can aid in the diagnosis and prognosis of these diseases. The present work can contribute to better understand the impedance of the TM when different forms of OME are present.

The study reported here shows a numerical analysis of a common ear disease. When recurrent, this pathology can cause hearing losses, which can lead to complicated social situations, especially for children that are in the critical period of language acquisition. When OME is not resolved, it can evolve to more complicated pathologies such as chronic otitis, which can have irreversible sensorineural consequences.

\section{Disclosure statement}

No potential conflict of interest was reported by the authors. 


\section{Funding}

This work was supported by Ministério da Ciência, Tecnologia e Ensino Superior, FCT, Portugal [grant number IF/00159/ 2014]; the SciTech - Science and Technology for Competitive and Sustainable Industries [project number UID/EMS/50022/ 2013], [project number NORTE-01-0145-FEDER-000022 ], cofinanced by Programa Operacional Regional do Norte (NORTE2020), through Fundo Europeu de Desenvolvimento Regional (FEDER).

\section{References}

American Academy of Family Physicians. 2004. Otitis media with effusion. Pediatrics. 113(5):1412-1429.

Areias B. 2014. Simulação biomecânica do ouvido humano, incluindo patologias do ouvido médio [master's thesis]. Porto: Faculdade de Engenharia da Universidade do Porto.

Atkinson H, Wallis S, Coatesworth AP. 2015. Otitis media with effusion. Postgrad Med. 127(4):381-385. ISSN 0032-5481. doi: 10.1080/00325481.2015.1028317.

Daniel HJ, Schmidt RT, Fulghum RS, Ruckriegal L. 1988. Otitis media: a problem for the physical anthropologist. Am J Phys Anthropol. 31(S9):143-167. ISSN 1096-8644. doi: 10.1002/ajpa.1330310508.

Douglas Swarts J, Bluestone CD. 2003. Eustachian tube function in older children and adults with persistent otitis media. Int J Pediatr Otorhinolaryngol. 67(8):853-859. ISSN 01655876. doi: 10.1016/S0165-5876(03)00127-7.

Doyle WJ. 1984. Functional eustachian tube obstruction and otitis media in a primate model: a review. Acta Otolaryngol. 98(Suppl 418):52-57. ISSN 0001-6489.

Doyle WJ, Swarts J, Banks J, Casselbrant ML, Mandel EM, Alper CM. 2013. Sensitivity and specificity of eustachian tube function tests in adults. JAMA Otolaryngol Head Neck Surg. 139(7):719-727. ISSN 2168-6181. doi: 10.1001/jamaoto.2013.3559.

Ferris P, Prendergast PJ. 2000. Middle-ear dynamics before and after ossicular replacement. J Biomech. 33(5):581-590. ISSN 0021-9290. doi: 10.1016/S0021-9290(99)00213-4.

Gan RZ, Dai CK, Wood MW. 2006. Laser interferometry measurements of middle ear fluid and pressure effects on sound transmission. J Acoust Soc Am. 120(6):3799-3810. ISSN 0001-4966. doi: 10.1121/1.2372451.
Gan RZ, Wang X. 2007. Multifield coupled finite element analysis for sound transmission in otitis media with effusion. J Acoust Soc Am. 122(6):3527-3538. ISSN 0001-4966. doi: $10.1121 / 1.2793699$.

Gentil F. 2008. Estudo biomecânico do ouvido médio [PhD thesis]. Porto: Universidade do Porto.

Gentil F, Parente M, Martins P, Garbe C, Jorge RN, Ferreira A, Tavares JMRS. 2011. The influence of the mechanical behaviour of the middle ear ligaments: a finite element analysis. Proc Inst Mech Eng H. 225(1):68-76. doi: 10.1243/09544119jeim783.

Hibbit D, Karlsson B, Sorenson P. 2004. ABAQUS analysis user's manual, version 6.5. Hibbit, Karlsson \& Sorensen, Inc.

Jazar RN. 2012. Advanced vibrations : a modern approach. 1st ed. New York (NY): Springer. ISBN 9781461441595.

Lee C-F, Chen P-R,Lee W-J, Chen J-H, Liu T-C. 2006. Threedimensional reconstruction and modeling of middle ear biomechanics by high-resolution computed tomography and finite element analysis. Laryngoscope. 116(5):711-716. ISSN 1531-4995.

Liu Y, Li S, Sun X. 2009. Numerical analysis of ossicular chain lesion of human ear. Acta Mech Sinica. 25(2):241-247. ISSN 0567-7718.

Prendergast PJ, Ferris P, Rice HJ, Blayney AW. 1999. Vibroacoustic modelling of the outer and middle ear using the finite-element method. Audiol Neurotol. 4:3-4.

Ravicz ME, Rosowski JJ, Merchant SN. 2004. Mechanisms of hearing loss resulting from middle-ear fluid. Hear Res. 195(1-2):103-130. ISSN 0378-5955. doi: 10.1016/j.heares.2004.05.010.

Sun Q, Gan RZ, Chang KH, Dormer KJ. 2002. Computerintegrated finite element modeling of human middle ear. Biomech Model Mechanobiol. 1(2):109-122. ISSN 16177959. doi: 10.1007/s10237-002-0014-z.

Swarts JD, Casselbrant ML, Teixeira MS, Mandel EM, Richert BC, Banks JM, El-Wagaa J, Doyle WJ. 2014. Eustachian tube function in young children without a history of otitis media evaluated using a pressure chamber protocol. Acta Otolaryngol. 134(6):579-587. doi: 10.3109/00016489.2014.882017.

Valente MH, de Ulhôa Escobar AM, Josefina S, Grisi FE. 2010. Aspectos diagnósticos da otite média com derrame na faixa etária pediátrica. Rev Bras Saúde Mater Infant. 10:157-170. ISSN 1519-3829.

Voss SE, Rosowski JJ, Merchant SN, Peake WT. 2000. Acoustic responses of the human middle ear. Hear Res. 150:43-69. doi: 10.1016/S0378-5955(00)00177-5.

Wang H, Merchant SN, Sorensen MS. 2007. A downloadable three-dimensional virtual model of the visible ear. ORL J Otorhinolaryngol Relat Spec. 69(2):63-67. ISSN 0301-1569. doi: 10.1159/000097369. 